\title{
IL PENSIERO ECONOMICO DELL'ILLUMINISMO CIVILE: UN PROGRAMMA DI RICERCA
}

\author{
PIER LUIGI PORTA(*) e ROBERTO SCAZZIERI(**)
}

L'Istituto Lombardo Accademia di Scienze e Lettere promosse nei giorni 13 e 14 dicembre 2011 un Convegno di studio sul pensiero economico dell' illuminismo lombardo che ebbe luogo nella sede dell'Istituto stesso nel Palazzo di Brera a Milano. Come è ampiamente riconosciuto, l'illuminismo lombardo costituisce uno dei momenti più alti e significativi del Settecento riformatore in Europa. Insieme con l'illuminismo napoletano esso rappresenta uno dei contributi più incisivi della cultura italiana allo sviluppo di una moderna tradizione europea di diritti e riforme civili.

Il Convegno di studio, del quale qui si pubblicano la più parte dei contributi in versione rivista, ha inteso mettere l'accento soprattutto sull'apporto creativo della scienza nuova dell'economia politica (o economia pubblica, o economia civile) alla cultura del Caffè e dei circoli verriani, che sono il cuore dell'illuminismo lombardo.

L'illuminismo lombardo fa proprio l'interesse dell'illuminismo francese per un'attività razionale di governo unendolo all'enfasi, tipica dell'illuminismo scozzese, posta sulle configurazioni e le dinamiche storiche della società civile. Tuttavia, esso si distingue da entrambi per l'attenzione rivolta alle connessioni fra legislazione, riforme e cultura civile. Si distingue da essi anche per essere parte del complesso intreccio di

(*) Istituto Lombardo Accademia di Scienze e Lettere; Università degli Studi, Milano-Bicocca.

${ }^{(* *)}$ Istituto Lombardo Accademia di Scienze e Lettere; Università degli Studi, Bologna. 
reciproche influenze che caratterizza il microcosmo degli stati e città italiane nel Settecento.

L'illuminismo lombardo è un illuminismo civile ed economico al tempo stesso: esso assume la socievolezza come punto di partenza ma considera la stessa come intrinsecamente connessa a tradizioni civili (reali o immaginate), mentre la trova espressa in modo prioritario attraverso la divisione del lavoro e le interdipendenze fra mercati. Alcuni dei più importanti contributi dell'Illuminismo lombardo studiano gli assetti economici e le disposizioni amministrative di una società che si governa attraverso centri autonomi di formazione e realizzazione delle decisioni. In questa prospettiva, economia, giustizia e legislazione acquistano una posizione fondamentale, e si evitano sia l'enfasi degli illuministi scozzesi sulla conformità spontanea in una società commerciale sia quella degli illuministi francesi sul disegno razionale delle politiche economiche e amministrative. ${ }^{1}$ Il coordinamento sociale è preso in esame a partire dagli ordinamenti civili esistenti e attraverso la griglia interpretativa fornita da alcuni prototipi di filosofia politica e morale.

Tutto questo conduce ad una caratteristica combinazione di ambizioni teoriche e preoccupazioni pratiche e spiega l'interesse degli illuministi lombardi per questioni come il mercato concorrenziale, le regole e gli interventi sull'organizzazione dell'agricoltura e delle manifatture, l'imposta fondiaria, la politica monetaria. ${ }^{2}$ Come ebbe a notare Franco Venturi, la tensione fra utopia e riforma è centrale nell'Illuminismo italiano e spiega la maggior parte dei suoi contributi più significativi e incisivi. ${ }^{3}$ In questa prospettiva, l'Illuminismo lombardo certamente si distingue soprattutto per il suo carattere pragmatico e per la sua attenzione per gli assetti amministrativo-costituzionali. ${ }^{4}$

Obiettivo centrale del Convegno di studio è stato quello di mettere a fuoco la relazione fra teoria economica e politiche riformatrici in

1 Questi aspetti sono considerati, rispettivamente, in Hont e Ignatieff (1983) e Rothschild (2001).

2 Su questi aspetti si possono vedere diversi contributi degli scriventi (Porta e Scazzieri, 1996, 1999, 2002a, 2002b) nonché, fra gli altri, Groenewegen, 1999, Hotta, 1999, Porta, 1990, Quadrio Curzio e Scazzieri, 1992, 2008.

3 Si vedano Venturi, 1969, 1970.

4 Questi aspetti sono presi in esame in diversi saggi di Alberto Quadrio Curzio (2007), oltre che nel volume Quadrio Curzio (1996). 
campo economico, giuridico e amministrativo. In questo ambito, l'Illuminismo lombardo è all'origine di due fra i più importanti contributi del Settecento riformatore italiano: la critica del sistema delle pene di Cesare Beccaria e le indagini di Pompeo Neri sui presupposti catastali della tassazione fondiaria. In entrambi i casi, un forte e originale impulso intellettuale è seguito da riforme istituzionali sia in Lombardia sia altrove. In particolare la Toscana e Parma saranno i primi stati europei ad abolire la pena di morte, mentre il nuovo sistema di imposizione della terra, introdotto in Lombardia anche per effetto delle proposte di Neri, esercita profonda influenza sulle riforme catastali in Inghilterra e in Francia.

Il volume si divide in due parti. La prima parte ('L'Illuminismo lombardo e il pensiero economico') con i contributi di Roberto Scazzieri ('L'illuminismo delle riforme civili: commercio, divisione del lavoro, produzione della ricchezza'), André Tiran ('Pietro Verri et JeanBaptiste Say: valeur, monnaie et loi des débouchés'), Manuela Albertone e Cecilia Carnino (" "Lusso di ostentazione" e "lusso di comodo. Tra economia e politica: un linguaggio di riforma della società nella Milano del "Caffè" '), Christine Lebeau ("Vers la construction d'une science administrative au XVIIIe siècle. L'example du cadastre de Milan'), Giuseppe Bognetti ('Governo dell'economia e teoria della politica economica'), Angelo Moioli ('Tariffe, dazi e politiche di commercio'), Alberto Quadrio Curzio e Roberto Scazzieri ('La teoria monetaria dell'illuminismo lombardo') tocca temi principalmente di teoria economica tanto sotto il profilo teorico quanto rispetto a talune applicazioni di particolare rilievo nel contesto storico dell'epoca teresiana in senso lato. In apertura di questa parte del volume, il lavoro di Roberto Scazzieri esamina e approfondisce le coordinate concettuali del programma di ricerca di cui il Convegno è stato una prima realizzazione. Nella seconda parte - con i saggi di Carlo Capra ('Il contesto sociale e politico dell'Illuminismo lombardo'), Sophus A. Reinert ("Guerra senza sangue' e l'aroma dei lumi: La cultura del Caffè tra politica e commercio internazionale nella Lombardia austriaca'), Giampaolo Massetto ('Beccaria tra diritto penale ed economia pubblica'), Germano Maifreda ('Sapere economico e metodo scientifico nell'Illuminismo lombardo: note da "Il Caffè"), Luigino Bruni ('Virtù civili e ricompense nell'Illuminismo europeo: Giacinto Dragonetti oltre l'Illuminismo lombardo') - il volume prende in considerazione spunti, 
influenze e sviluppi nei quali prevalgono aspetti multidisciplinari. Questo al fine di collocare in contesto l'impostazione economica della Scuola lombarda, che non è mai (come invece in parte accade in Francia) incline a decretare la superiorità astratta del ragionamento economico per sé preso, ma si fonda invece sulla capacità di colloquio e scambio tra diverse discipline e diverse modalità di analisi della società secondo un punto di vista che risente della lezione dell'economista napoletano Antonio Genovesi, da tutti riconosciuto come lo studioso all'origine degli studi di economia politica ('economia civile') in Italia. In conclusione del volume, il saggio di Pier Luigi Porta ('Nuove prospettive negli studi economici sull'illuminismo lombardo') si propone di raccogliere, in forma indiretta, gli spunti offerti dal Convegno a dimostrazione del contributo fondamentale dell'Illuminismo lombardo durante il periodo di transizione dalla Fisiocrazia ad Adam Smith.

La lezione dell'illuminismo economico lombardo è essenziale per comprendere i temi che animano il dibattito economico nel nostro paese, in Europa e nel più ampio contesto internazionale. In questa prospettiva intendiamo promuovere, in collegamento con l'Istituto Lombardo, la più ampia collaborazione fra studiosi per approfondire la conoscenza di contributi il cui tratto più caratteristico è stato la consapevolezza che riforme efficaci e politiche incisive richiedano in ogni caso una precisa identificazione storico-analitica di quello che Giandomenico Romagnosi descriverà come 'ordine sociale delle ricchezze' (Romagnosi, 1827, p. 24).

I curatori di questo volume esprimono un vivo ringraziamento a Maria Cristina Bacchi per il contributo bibliografico essenziale nella preparazione dell'indice dei nomi. 


\section{BIBLIOGRAFIA}

Groenewegen, P. (1999) 'The Significance of Verri's Meditazioni in the History of Economic Thought: the Wider European Influences', in C. Capra (a cura di),. Pietro Verri e il suo tempo, Bologna, Cisalpino. Istituto Editoriale Universitario, vol. II, pp. 693-708.

Hont, I. e Ignatieff, M. (1983) (a cura di) Wealth and Virtue. The Shaping of Political Economy in the Scottish Enlightenment, Cambridge, Cambridge University Press.

Hotta, S. (1999) 'European Sources of Pietro Verri's Economic Thought', in C. Capra (a cura di),. Pietro Verri e il suo tempo, Bologna, Cisalpino. Istituto Editoriale Universitario, vol. II, pp.709-26.

Porta, P.L. (1990) 'Le lezioni di economia di Cesare Beccaria', in S. Romagnoli e G.D. Pisapia (a cura di), Cesare Beccaria tra Milano e l'Europa, Roma-Bari, CariploLaterza, pp. 356-70.

Porta, P.L. e Scazzieri, R. (1996) 'Concorrenza e società civile', in A. Quadrio Curzio, a cura di, Economia e Istituzioni: il paradigma lombardo tra $i$ secoli XVIII e XIX, Bologna, Il Mulino, pp. 15-58.

Porta, P.L. e Scazzieri, R. (1999) 'Il contributo di Pietro Verri alla teoria economica. Società commerciale, società civile e governo dell'economia', in C. Capra (a cura di), Pietro Verri e il suo tempo, Bologna, Cisalpino. Istituto Editoriale Universitario, vol. II, pp.813-52.

Porta, P.L. e Scazzieri, R. (2002a) 'Pietro Verri's Political Economy: Commercial Society, Civil Society, and the Science of the Legislator', History of Political Economy, 34 (1, Spring), pp. 83-110.

Porta, P.L. e Scazzieri, R. (2002b) 'Political Economy in Italy. Competition and Civil Society in the Milanese school, 1750-1850', in M. Psalidopoulos e M.E. Mata (a cura di), Economic Thought and Policy in Less Developed Europe, London e New York, Routledge, pp. 187-210.

Quadrio Curzio, A. (2007) Economisti ed economia. Per un'Italia europea: paradigmi tra il XVIII e il XX secolo, Bologna, Il Mulino.

Quadrio Curzio, A. (1996) (a cura di) Economia e istituzioni: Il paradigma lombardo tra i secoli XVIII e XIX, Bologna, Il Mulino.

Quadrio Curzio, A. e Scazzieri, R. (1992) 'Dall'economia politica al governo dell'economia: riflessioni sul contributo di Cesare Beccaria e Pietro Verri sulla teoria e pratica della moneta', in N. Acocella, G.M. Rey e M. Tiberi (a cura di), Saggi di politica economica in onore di Federico Caffè, vol. II, Milano, Franco Angeli, pp. 141-81.

Quadrio Curzio, A. e Scazzieri, R. (2008) 'Historical Stylisations and Monetary Theory', in R. Scazzieri, A.Sen e S. Zamagni (a cura di) Markets, Money and 
Capital. Hicksian Economics for the Twenty-First Century, Cambridge, Cambridge University Press, pp. 185-203.

Romagnosi, G. (1827) 'Quesito. Il modo usato da alcuni scrittori di oggidì nel trattare le Dottrine economiche è forse plausibile?', Annali Universali di Statistica, 13, pp. 23-30.

Rothschild, E. (2001) Economic Sentiments. Adam Smith, Condorcet and the Enlightenment, Cambridge, Mass.; London, Harvard University Press.

Venturi, F. (1969) Settecento riformatore. Da Muratori a Beccaria, Torino, Einaudi.

Venturi, F. (1970) Utopia e riforma nell'illuminismo, Torino, Einaudi. 